\title{
BMJ Open Psychosocial and health behavioural impacts of COVID-19 pandemic on adults in the USA: protocol for a longitudinal cohort study
}

\author{
Hoda Badr (D , , Abiodun Oluyomi, ${ }^{1}$ Maral Adel Fahmideh, ${ }^{1,2}$ Syed Ahsan Raza, ${ }^{1}$ \\ Xiaotao Zhang (i) , ${ }^{1,3}$ Ola El-Mubasher, ${ }^{1}$ Christopher Amos ${ }^{1}$
}

To cite: Badr H, Oluyomi A, Adel Fahmideh M, et al. Psychosocial and health behavioural impacts of COVID-19 pandemic on adults in the USA: protocol for a longitudinal cohort study. BMJ Open 2020;10:e044642. doi:10.1136/ bmjopen-2020-044642

- Prepublication history for this paper is available online. To view these files, please visit the journal online (http://dx.doi org/10.1136/bmjopen-2020044642).

Received 08 September 2020 Revised 07 November 2020 Accepted 26 November 2020

Check for updates

(C) Author(s) (or their employer(s)) 2020. Re-use permitted under CC BY-NC. No commercial re-use. See rights and permissions. Published by BMJ.

${ }^{1}$ Section of Epidemiology and Population Sciences, Department of Medicine, Baylor College of Medicine, Houston,

Texas, USA

${ }^{2}$ Center for Epidemiology and Population Health, Department of Pediatrics, Baylor College of Medicine, Houston, Texas, USA ${ }^{3}$ Epidemiology, University of Texas MD Anderson Cancer Center, Houston, Texas, USA

Correspondence to

Dr Hoda Badr;

hoda.badr@bcm.edu

\section{ABSTRACT}

Introduction Although social distancing may help contain the spread of COVID-19, the social isolation and loneliness it causes can heighten stress, contribute to unhealthy lifestyle behaviours and have deleterious effects on social relationships. This ongoing longitudinal cohort study aims to (1) characterise the psychological, social and health behavioural impacts of the COVID-19 pandemic over a 12-month period in the USA; (2) determine whether these impacts differ for certain subgroups based on sociodemographics and other individual-level factors; and (3) explore whether there are modifiable factors (eg, coping, social support) that moderate the effects of the pandemic over time.

Methods and analysis Adults (aged $\geq 18$ years) who were fluent in either English or Spanish were recruited via social media and invited to complete an online survey during the 8-week period from 13 April to 8 June 2020 (baseline). Follow-up surveys will be conducted 6 and 12 months after baseline. Data transformations, non-parametric tests or other alternative methods will be used when appropriate. Descriptive statistics and cross-sectional analyses will be performed. Longitudinal associations will be analysed using multilevel modelling with time-variant and time-invariant predictors of change in trajectory over the study period.

Ethics and dissemination Research ethics approval was received from the Baylor College of Medicine Institutional Review Board (H-47505). Overall, this study will provide timely information that can be used to inform public health messaging strategies and guide development of assessment tools and interventions to support vulnerable individuals dealing with the long-term impacts of the COVID-19 pandemic.

\section{INTRODUCTION}

On 31 December 2019, China reported to the WHO that four cases of pneumonia with an unknown cause had been identified in Wuhan, Hubei Province. ${ }^{1}$ The cause was discovered to be a novel coronavirus, SARS-CoV-2, and the ensuing outcome of the infection began to be called COVID-19. Person-to-person transmission was subsequently confirmed, and
Strengths and limitations of this study

- This study will provide timely information on the psychosocial and health behavioural impacts of the COVID-19 pandemic, including the evolving response of COVID-19 preventive behaviours (eg, social distancing, hand hygiene, mask wearing).

- A key strength is the repeated measures design, which will facilitate the tracking of changes in mental health and health behaviours over time.

- This study will recruit an online convenience sample. As with all non-probability samples, there is limited ability to generalise findings to the populations from which participants were drawn.

- Online recruitment methods can be limited by demographic representation. To overcome this, we will use paid social media advertising and crowdsourcing survey platforms to recruit a national study sample that is sociodemographically diverse.

- A limitation of this study is the focus on adults living in the USA; thus, study findings may not generalise to adults living in other countries.

the virus began to spread. ${ }^{2}{ }^{3}$ Owing to the absence of a vaccine and the lack of effective pharmacological interventions, social distancing became the primary mitigation strategy. Social distancing is a set of measures intended to prevent the spread of a contagious disease by increasing the space between individuals and decreasing the frequency of contact. ${ }^{45}$ It can be implemented in an individual (eg, avoiding physical contact), group (eg, cancelling group activities where individuals will be in close contact) and/or community level (eg, instructing people to stay at home and shutting down non-essential businesses). In response to the unprecedented threat to public health caused by COVID-19, China became the first country to mandate social distancing by instituting a strict quarantine on 23 January 2020 in Hubei and the surrounding provinces. ${ }^{6}$ However, by that 
time, the virus had already spread outside China. French health officials confirmed the first three cases in Europe on 24 January, and the first case of person-to-person transmission was confirmed in the USA on 30 January. $^{7}$ On $11 \mathrm{March}$, the number of cases in the USA surpassed $1000{ }^{7}$ and the WHO declared COVID-19 a worldwide pandemic. ${ }^{8}$ Shortly thereafter, on 13 March, the president of the USA declared COVID-19 to be a national emergency. ${ }^{9}$

Faced with an exponential rise in cases and deaths and in an effort to avoid overwhelming healthcare systems, countries around the world began to shut down their economies and severely restrict public movement. By 26 March, the USA surpassed China and Italy to become the world's most infected nation with 83836 cases, ${ }^{7}$ and by early April, 42 US states had declared state-wide stayat-home orders. ${ }^{10}$ US states that did not institute stayat-home orders were Arkansas, Iowa, Nebraska, North Dakota, Oklahoma, South Dakota, Utah and Wyoming. By the end of the first week in June, rates of infection in the USA had begun to slowly decline, stay-at-home orders in most states had expired and 34 states had either reopened or were in the process of a phased, state-wide reopening. ${ }^{10}$ In addition, governors in eight hard-hit states had allowed counties or regions that met criteria for slowing the outbreak to open (California, Illinois, Michigan, New York, Oregon, Pennsylvania, Tennessee and Washington). ${ }^{10}$

Although the disruption of everyday life and social networks may be necessary to contain transmissible diseases such as COVID-19, social distancing challenges the deeply rooted human need to connect with others. ${ }^{11}$ Social connection helps people regulate negative emotions, cope and remain resilient in times of stress. ${ }^{12}{ }^{13}$ By contrast, loneliness and social isolation can worsen the burden of stressful circumstances, contribute to unhealthy lifestyle behaviours (eg, smoking, drinking alcohol) ${ }^{1415}$ and have deleterious effects on mental health, romantic relationships and physical health. ${ }^{16-18}$ Emerging research suggests that the mental health impacts of COVID-19 vary widely, ${ }^{19-21}$ with sociodemographic factors accounting for some of this variation. For example, studies from the USA ${ }^{22} 23$ and other countries $^{24-34}$ have shown that women, younger adults, those with lower levels of education and/or income and those with a pre-existing mental or chronic health condition are more vulnerable to the adverse mental health effects of the pandemic. Informal family caregivers may be especially vulnerable to the effects of reduced access to healthcare, social services and resources that are central to the management of their/their loved one's condition, but research on the effects of the pandemic on their emotional well-being has been relatively limited. ${ }^{35}$ Similarly, data from the US Centers for Disease Control and Prevention suggest that racial/ethnic minority communities are disproportionately affected by COVID19 , but few studies have enrolled sufficient numbers of minorities to examine differential mental health or health behavioural impacts. Finally, despite the fact that strict stay-at-home and social distancing measures have resulted in families and romantic partners either being confined together in their homes or sequestered from one another to reduce exposure risk, few studies have been published on the relationship impacts of COVID-19. ${ }^{36}$ Just as it is possible that the disruptions of daily routines, reduced social outlets and reduced physical contact caused by the pandemic may contribute to decrements in physical, psychological and/or relationship well-being, it is also possible that decrements in one of these domains could adversely affect functioning in other domains, resulting in a cascade effect. Thus, beyond understanding individual risk/protective factors, more research is needed on how different domains of well-being are affected by the pandemic and how they affect each other over time.

To address these knowledge gaps, we are currently conducting a population-based survey to simultaneously examine the psychological, social and health behavioural impacts of the COVID-19 pandemic on individuals living in the USA. Study objectives are to (1) characterise the psychological, social and health behavioural impacts of the COVID-19 pandemic during the mandatory stayat-home order period in the USA (baseline) and 6 and 12 months later; (2) determine whether psychological, social and behavioural impacts differ for certain subgroups based on sociodemographics (eg, gender, race/ethnicity), geography, health status and caregiving responsibilities; and (3) explore whether there are modifiable factors that moderate effects of the pandemic over time (eg, individual and/or communal coping, social support) that could be targeted to enhance psychosocial and behavioural adjustment.

\section{METHODS AND ANALYSES \\ Study design}

Given the rapidly evolving nature of the COVID-19 pandemic and varied response across different states and municipalities across the USA, baseline surveys were administered during an 8-week recruitment window (13 April 2020 to 8 June 2020), which corresponded to the initial stay-at-home order period in most of the USA. ${ }^{10}$ Follow-up surveys will be administered 6 and 12 months after baseline to examine overtime effects.

\section{Patient and public involvement}

Given the ongoing pandemic, it was not appropriate or possible to involve patients or the public in the design, or conduct, or reporting, or dissemination plans of our research.

\section{Eligibility}

Eligible individuals are those $\geq$ aged 18 years, who reside in the USA and are fluent in English or Spanish. 


\section{Sampling strategy and recruitment}

Our goal was to recruit a minimum of 2000 participants at baseline. As return rates for online health-related surveys are approximately $50 \%,{ }^{37}$ we hope to obtain 6 -month follow-up data from 1000 baseline survey respondents and 12-month follow-up data from 500 baseline survey respondents.

A range of methods were used to target specific groups to increase the representativeness of the sample. Baseline study participants were initially recruited via unpaid social media advertisements. These strategies included a press release, internal Baylor College of Medicine (BCM) communications (eg, electronic newsletters) and posts about the study on the principal investigator's ( $\mathrm{HB}$ ) lab page and on social media accounts (eg, LinkedIn, Twitter, Reddit). We also made electronic contact with colleagues, established interest groups and community advocates, and requested that they endorse our project by posting the project advertisement so that it was visible to their contacts/group members. To recruit survey respondents from all 50 US states and ensure a more representative sample with respect to gender and ethnic/racial composition, we also pursued targeted online advertising via Facebook sponsored posts and an online survey crowdsourcing platform, Soapbox Sample (https://www.soapboxsample. $\mathrm{com} /$ ). Crowdsourcing platforms are web-based marketplaces that allow researchers to post research tasks (such as surveys) that interested subjects can complete for payment or other incentives. Crowdsourcing offers an easy way to quickly recruit a large number of respondents, ${ }^{38}$ and platforms such as Soapbox Sample draw a broad demographic of workers who can meet even very specific and targeted inclusion criteria, ${ }^{394}$ making this an appealing option for recruiting hard-to-reach groups. ${ }^{41}$

\section{Procedures}

\section{Baseline survey}

Because this was an online survey, we obtained a waiver of written informed consent under Department of Health and Human Services (DHHS) regulations at 45 CFR 46.117(c) and the Common Rule. The advertisements and social media posts used for participant recruitment contained a web hyperlink that directed participants to the survey website. The survey landing page contained a brief cover letter describing the purpose of the research, eligibility criteria and a plain language statement. If, after reading the study description, individuals were interested in participating, they were asked to check a box that confirmed that they had read the Plain Language Statement, that they understood its contents and that they consented to participate in the study.

The survey was administered online in English and Spanish on the Qualtrics survey platform (Provo, Utah, USA) ${ }^{42}$ Given that accountability and validity can be more difficult to enforce in online research, ${ }^{39}{ }^{43}$ we used tactics to help prevent machine responses (eg, Captcha). We also prevented participants from taking the survey more than once by using the Qualtrics 'Prevent Ballot Box Stuffing' feature. Once accessed, participants could take up to 2 weeks to complete the survey. On completion of the baseline survey, all participants were assigned a unique subject ID number and asked to provide their email contact information if they would like to be contacted for the follow-up surveys. The subject ID number will be embedded in the follow-up surveys so that we can link these data to respondents' baseline survey responses.

\section{Follow-up surveys}

Participants will be recontacted 6 and 12 months after completion of the baseline survey via email invitation. Regardless of whether a participant completes the 6-month follow-up survey, he/she will receive the invitation to complete the 12-month survey. All emails to participants will contain a link to opt out from survey completion or from the study entirely.

\section{Measures}

Table 1 provides an outline of the survey measures. Measures include (1) sociodemographic questions (eg, age, gender, race/ethnicity, marital status, education, household income, zip code and cross streets), (2) medical variables (eg, COVID-19 status, exposures, symptoms, testing, chronic/serious health conditions, psychiatric diagnoses and effects of the pandemic on disease self-management), (3) caregiving status and experiences, (4) mental health impacts, (5) social/relationship impacts, (6) health behavioural impacts with regard to COVID-19 preventive behaviours (eg, adherence to stay-at-home orders, social distancing, hand hygiene and mask wearing), (7) lifestyle behavioural impacts (eg, alcohol drinking, tobacco use, vaping, exercise and diet), (8) other impacts (eg, different aspects of life affected by COVID-19 and sleep) and (9) possible explanatory variables including knowledge, individual and dyadic coping strategies, and social connectedness and social support. Based on zip code and cross street information, we will use the 2010 rural-urban commuting area (RUCA) codes to define urban and rural areas of residence. ${ }^{44-46}$ RUCA codes classify census tracts based on measures of population density, urbanisation and daily commuting flows. To examine regional variation, states of residence will be divided into one of the four major US census regions: Northeast, South, Midwest and West.

\section{Analytical approach}

\section{Quantitative data}

Survey data will be exported from Qualtrics to IBM SPSS Statistics for Windows V.26 (IBM) for data cleaning and analysis. We will monitor participant retention to gauge the amount of missing data and take proactive steps to minimise it. If necessary, we will use the restricted maximum likelihood method for estimating the longitudinal statistical models to minimise the impact. This method will allow us to retain cases with partial data with a less restrictive assumption: missing at random (MAR). We will use Little's missing completely at random (MCAR) 
Table 1 Survey measures

\begin{tabular}{ll} 
Construct & Measure \\
\hline $\begin{array}{l}\text { Sociodemographics (20 } \\
\text { items) }\end{array}$ & $\begin{array}{l}\text { Age, gender, race/ethnicity, marital status, current living arrangement, number of people } \\
\text { living in household, relation, education, employment status, changes in employment due to } \\
\text { COVID-19, household income, zip code and cross street }\end{array}$
\end{tabular}

\section{Medical variables}

COVID-19 status, exposures, Adapted from the CoRonavlruS Health Impact Survey V.0.357; Do you think you may have had symptoms and testing $(10$ items)

Health conditions and selfmanagement (11 items)

COVID-19 but did not get tested for any reason? If yes, please specify (open response)

Diagnosis of cancer (type, stage, treatment) or other chronic/serious medical conditions that require medical management at home; treatment delays due to COVID-19; How has COVID-19 affected daily management of your condition (open-ended)? What are your biggest concerns regarding your health/healthcare since COVID-19?

\section{Caregiving status and} experiences (38 items)

Since the beginning of the pandemic, have you provided care for someone in your household who had COVID-19 (1=yes, 2=no), cancer ( $1=y e s, 2=n o)$, a chronic/serious condition that required medical management at home (1=yes (specify), $2=$ no) or someone with a cognitive, physical or intellectual disability ( $1=y e s$ (specify), $2=n o$ )? What is their relationship to you? Nature and extent of caregiving activities; change in caregiving responsibilities as a result of COVID-19; How has COVID-19 affected daily management of your loved one's condition (open response)? Is there someone who shares caregiving responsibilities with you (1=yes (specify), $2=$ no)? Caregiving challenges experienced as a result of COVID-19 (open response); unmet information, resource and support needs as a result of COVID-19 (open response); concerns about keeping the person you are caring for safe from COVID-19 (open response)

\section{Mental health impacts}

COVID-19 Stress (9 items)

Top 5 COVID-19 stressors (open response); Perceived Stress Scale-4-item Short Form ${ }^{58}$

General depression (4 items)

PROMIS Depression 4-item Short form ${ }^{59}$

General anxiety (4 items) PROMIS Anxiety 4-item Short Form ${ }^{59}$

Psychosocial Impact of COVID-19 (9 items)

PROMIS Psychosocial IIIness Impact-Negative Scale (instructions reworded to refer to before and after COVID-19 pandemic); positive impacts/silver linings of COVID-19 pandemic (open response)

\section{Social/relationship impacts}

Relationship changes due to Relationship satisfaction, conflict and criticism before and since COVID-19 COVID-19 (6 items)

Social isolation (5 items) Social isolation items from the Nottingham Health Profile $^{60}$

\section{Health behaviour impacts-COVID-19 preventive measures}

Adherence to stay-at-home orders (5 items)

Does the area where you live have a stay-at-home order (1=yes, 2=no, 3=I don't know)? To what extent do you currently follow the stay-at-home order ( $0=$ not at all to $10=$ completely)? How often did you leave your home in the past week, did you leave your home yesterday? Reasons. How many people did you come into contact with when you left your home yesterday?

Social distancing (10 items)

Degree of adherence to social distancing, attitudes and beliefs about the effectiveness of social distancing for stopping spread of COVID-19, perceived barriers and motivations for social distancing

Hand hygiene Frequency of handwashing and hand sanitiser use, and beliefs about the effectiveness of hand (3 items) hygiene for stopping spread of COVID-19

Mask use Use of face coverings, perceived barriers and motivations for mask use, and beliefs about the

(18 items) effectiveness of mask use for stopping spread of COVID-19

Other measures ( 1 item)

What other steps have you taken to keep yourself and other members of your household safe from COVID-19?

\section{Health behaviour impacts - lifestyle behaviours}

Alcohol use

Items from AUDIT-C ${ }^{61}$; Has your drinking increased/decreased/stayed the same since

(4 items)

COVID-19? Since COVID-19, I have had trouble controlling my drinking (1=strongly disagree to $5=$ strongly agree) 
Table 1 Continued

\begin{tabular}{ll} 
Construct & Measure \\
\hline Tobacco use & Two items on current smoking status and type and number of tobacco products smoked per \\
(4 items) & $\begin{array}{l}\text { day, taken from the Global Adult Tobacco Survey }{ }^{62} \text {; Has your tobacco consumption increased/ } \\
\text { decreased/stayed the same since COVID-19? Are you worried about being at increased risk of }\end{array}$ \\
& COVID-19 due to smoking (1=yes, 2=no)?
\end{tabular}

Exercise (1 item)

Diet (2 items)

\section{Since COVID-19, I am exercising more ( 1 =strongly disagree to $5=$ strongly agree)}

Since COVID-19, I am eating more healthy foods; since COVID-19, I am snacking on more unhealthy foods ( $1=$ strongly disagree to $5=$ strongly agree)

\section{Other impacts}

Aspects of life affected by COVID-19

(12 items)

Sleep (4 items)

\section{Explanatory variables}

COVID-19 knowledge, sources Do you feel you know enough to keep yourself and others from getting COVID-19 (0=not at all of information and uncertainty (4 items) to $10=$ extremely)? Sources of information about COVID-19 (10 items); Mishel Uncertainty in Illness Scale-Short Form ${ }^{64}$

Degree to which COVID-19 has caused problems and/or resulted in positive changes with six different aspects of life (eg, work life, social life, home life, sex life, work-life balance and selfcare) on a scale of 0 (not at all) to 10 (extremely). PROMIS Sleep Disturbance $4 a^{63}$

Individual coping strategies (20 Brief COPE $^{65}$

items)

Dyadic coping strategies ( 20
items)

Social connectedness and social supports (6 items)
Stress communication (4 items), partner response to stress (9 items), common dyadic coping (4 items) and dyadic coping evaluation (2 items) sections of the Dyadic Coping Inventory ${ }^{66}$

How often in the past week did you spend time with someone who does not live with you? How do you keep in touch with others during the pandemic? How often in the past week did you talk to friends/relatives on the phone or video chat? Since the pandemic, how many individuals do you feel close to who live outside your household? Since the COVID-19 pandemic, how many individuals do you feel you can rely on for help if you needed something? Use of healthcare/community/government resources and supports.

AUDIT-C, Alcohol Use Disorders Identification Test-Concise; COPE, Coping Orientation to Problems Experienced; PROMIS, Patient Reported Outcome Measurement Information System.

test to determine the type of missing data. ${ }^{47}$ If the test is not significant, data will be treated as MCAR. To address minor amounts of missing data, items will be imputed and used to obtain point estimates. However, as this is a longitudinal study, missing data from attrition are more likely. Outcome missingness due to dropout (yes/no) will be examined using a hierarchical logistic regression model for clustered data using a generalised linear mixed model (LMM) procedure with a logit link function and random intercept. Sociodemographic and medical variables will be examined to identify factors that predict dropout.

For each instrument, recommended methods of computation and summarisation of scales and subscales will be followed. The variables will be assessed to determine distributional characteristics and ceiling/floor effects. In secondary analyses, model parameters and preliminary assessments of group differences will be estimated using LMMs. Trend analyses will indicate the shape of group trajectories over time and possible group-by-time interactions. Individual plots of participants' trajectories on major outcomes will be developed. ${ }^{48}$

When predicting study outcomes (eg, mental health, health behaviours), a random-effects $\mathrm{LMM}^{49}$ will be used. Full information maximum likelihood for parameter estimates does not assume patients are measured at all time points and will thus include participants with missing assessments, and in the case of MAR or MCAR will provide unbiased estimates and accurate SEs. ${ }^{50}$ If the MCAR assumption does not hold, we will use multiple imputation by using likelihood methods to estimate pattern mixture and selection models that condition on the baseline characteristics and latent variables to account for non-ignorable missing data.

Data analysis will be conducted in SPSS V.26, SAS V.9.4 or Stata/MP Software V.16. Study variables will be assessed to determine distributional characteristics and ceiling/ floor effects. Data transformations, non-parametric tests or other alternative methods will be used when appropriate. We will use descriptive statistics (ie, frequencies, means, SD) to characterise participant responses. We will also use $\chi^{2}$ tests (for dichotomous variables) or independent-samples t-tests (for continuous variables) to examine differences in the study outcome variables based on sociodemographics, health status and/or caregiving status. Non-parametric equivalents of these tests (ie, Fisher's exact test or the Wilcoxon Mann-Whitney test) will be used where appropriate. 
Cross-sectional and longitudinal analyses will be performed. For the former, multivariate linear or logistic regressions will be used, depending on the nature of the outcomes. For longitudinal analyses, LMM or logistic mixed model regressions will be used, depending on the nature of the outcomes, to account for repeated measurements. Where relevant, all associations will be investigated in unadjusted analyses and then in adjusted analyses-the latter controlling for the baseline effects of sociodemographic or health-related variables that are significantly associated with the outcome of interest. Decisions about the inclusion of specific covariates in each model will be made using directed acyclic graphs. ${ }^{51}$ In these models, we will regress an outcome (eg, anxiety) onto 'time', any moderator variables of interest and background covariates. Time will be treated as a continuous predictor in all models (with the baseline time point coded as 0,6 -month time point coded as 1 and 12-month time point coded as 2). The influence of potential moderators on the relationship between these associations will be investigated by including interaction terms.

\section{Power}

For available sample sizes of 500 participants, we can detect correlations of 0.05 or higher with $5 \%$ significance and $80 \%$ power among variables. With a sample size of 500 , we will have $80 \%$ power (two-tailed alpha $=5 \%$ ) to detect effects of proportional differences among groups of at least $11 \%$, assuming $\geq 20 \%$ of the sample falls in one group. We will be able to detect standardised effect differences of at least 0.31 standardised units if $\geq 20 \%$ of the sample falls in a smaller group.

\section{Qualitative analysis}

Text responses to open-ended questions will be transcribed and analysed thematically to determine common themes. Thematic analysis is a method of analysing qualitative data that is focused on identifying, examining and recording major patterns or themes in the data. ${ }^{52}$ We will follow the six-stage process described by Braun and Clarke ${ }^{52}$ for finding patterned responses or themes. (1) to familiarise ourselves with the data, all open-ended responses will be read by research team members (Dr Badr and two trained analysts) to obtain a sense of the breadth and depth of the data. Team members will meet to discuss initial impressions. (2) We will generate initial codes by extracting all relevant text units that relate to the question of interest and giving them a brief label that captures the essence of the text unit. The team will discuss, refine and verify all the codes and organise them in a data display table. (3) The team will then search for themes by dividing the codes into broad overarching themes based on code similarities. Visual representations will be developed to explore the relationships among codes within each of the themes. (4) Themes will be reviewed and revised by the team and organised into a coherent pattern. A coherent pattern includes internal homogeneity (ie, the codes link together meaningfully in each theme) and external heterogeneity (ie, there are clear distinctions between the themes). The team will then re-examine the narrative data set as a whole to ensure that all relevant data were captured by one of the themes. (5) Next, we will define and name themes and (6) produce a final report that provides a detailed account of each theme prepared. Disagreements will be resolved via mutual discussion, and an audit trail will be maintained to chronicle all methodological and analytical decisions.

\section{Recruitment progress}

We have completed baseline survey data collection. Among the 2435 respondents who consented to participate and accessed our survey during that time period, 2222 provided usable data, yielding a $91 \%$ overall survey completion rate. Five hundred and nineteen participants $(23 \%)$ completed less than $50 \%$ of the survey, 188 (9\%) completed $50 \%-80 \%$ and $1515(68 \%)$ completed $80 \%-100 \%$. For the 2222 who provided usable data, the median completion time was $24.39 \mathrm{~min}$ (mean $=1.67$ hours; $\mathrm{SD}=11.39$ hours; range $=5.08 \mathrm{~min}$ to 9.80 days). Follow-up survey data collection is ongoing.

\section{DISCUSSION}

Chronic stress and social isolation brought about by the COVID-19 pandemic present significant risks to the physical, psychological and social well-being of US adults. This project seeks to investigate the psychosocial and health behavioural impacts of the pandemic, including the evolving response of COVID-19 preventive behaviours (eg, social distancing, hand hygiene, mask wearing). The novel contribution of this study will be the repeated measures design, which will facilitate the tracking of changes in mental health and behaviours over time.

As with all non-probability samples, there is limited ability to generalise findings to the populations from which participants were drawn. ${ }^{53}$ In addition, online recruitment methods can be limited by demographic representation. ${ }^{54}$ To overcome this limitation, we used paid social media advertising and crowdsourcing survey platforms to recruit a national study sample that is sociodemographically diverse. These issues notwithstanding, we believe that use of an online convenience sample is appropriate for this study for three reasons. First, a limited budget prevents us from using more costly probability-based sampling methods. Second, the scale of the project (national survey) and the nature of the COVID-19 pandemic and stay-at-home orders render it highly impractical to recruit participants using traditional in-person or community-based approaches. ${ }^{55}$ Third, convenience samples are frequently used to recruit members of hard-to-reach populations (eg, socially isolated individuals) because reliable sampling frames are generally not available. ${ }^{56}$

This project was designed to elucidate health disparities and provide timely information to government, researchers and communities on the psychosocial and 
behavioural effects of the ongoing COVID-19 pandemic on US adults. Findings can be used in several ways, including (1) to guide development of assessment and screening tools to identify vulnerable individuals who are at greatest risk, (2) to guide researchers in developing targeted interventions to help support vulnerable individuals deal with the long-term impacts of the pandemic on their health and well-being and (3) to help policymakers develop public health messaging strategies and practical information and advice for the public regarding how to cope and protect themselves and others during the pandemic.

\section{ETHICS AND DISSEMINATION}

Research ethics approval for this study was received from the BCM Institutional Review Board (H-47505) with a waiver of written consent under DHHS regulations at 45 CFR 46.117(c) and the Common Rule. Findings will be disseminated through peer-reviewed publication and will be presented at conferences related to this field.

\section{Twitter Xiaotao Zhang @fishnytao}

Acknowledgements We would like to thank Navya Kumar and Lauren Greenwood who assisted with setting up the survey in Qualtrics and developing the study codebook. We would also like to thank Venus Gines who translated the survey and study advertisements to Spanish. Finally, we would like to thank all of the study participants for their involvement in the study during this very difficult and stressful time.

Contributors $\mathrm{HB}, \mathrm{AO}$ and $\mathrm{CA}$ contributed to the conceptualisation of the study, drafting of the study protocol and selection of survey items/measures. HB drafted the manuscript and together with OEM developed all study materials, including the online Qualtrics survey and study advertisements. AO, CA, SAR, XZ, MAF and OEM wrote or revised sections of the manuscript. All authors approved the final version of the manuscript.

Funding HB and CA's work on this study was supported by 0T2HL158258 (PI: Vishwantha). CA's work was also supported by Cancer Prevention Research Institute of Texas grant RR170048. MAF, SAR, XZ were supported by the Research Training Award for Cancer Prevention Post-Graduate Training Program in Integrative Epidemiology from the Cancer Prevention and Research Institute of Texas (grant number RP160097, PI: M. Spitz). This study was also supported by the facilities and resources of the Dan L Duncan Comprehensive Cancer Center P30 CA125123.

\section{Competing interests None declared.}

Patient and public involvement Patients and/or the public were not involved in the design, or conduct, or reporting, or dissemination plans of this research.

\section{Patient consent for publication Not required.}

Open access This is an open access article distributed in accordance with the Creative Commons Attribution Non Commercial (CC BY-NC 4.0) license, which permits others to distribute, remix, adapt, build upon this work non-commercially, and license their derivative works on different terms, provided the original work is properly cited, appropriate credit is given, any changes made indicated, and the use is non-commercial. See: http://creativecommons.org/licenses/by-nc/4.0/.

\section{ORCID iDs}

Hoda Badr http://orcid.org/0000-0002-4549-9111

Xiaotao Zhang http://orcid.org/0000-0002-3968-5030

\section{REFERENCES}

1 Phelan AL, Katz R, Gostin LO. The novel coronavirus originating in Wuhan, China: challenges for global health governance. JAMA 2020;323:709-10.
2 Li Q, Guan X, Wu P, et al. Early transmission dynamics in Wuhan, China, of novel coronavirus-infected pneumonia. N Engl J Med 2020;382:1199-207.

3 Lai C-C, Shih T-P, Ko W-C, et al. Severe acute respiratory syndrome coronavirus 2 (SARS-CoV-2) and coronavirus disease-2019 (COVID-19): the epidemic and the challenges. Int J Antimicrob Agents 2020;55:105924.

4 Glass RJ, Glass LM, Beyeler WE, et al. Targeted social distancing design for pandemic influenza. Emerg Infect Dis 2006;12:1671-81.

5 World Health Organization. Coronavirus disease 2019 (COVID-19): situation report, 72; 2020.

6 New York Times. Scale of China's Wuhan Shutdown Is Believed to Be Without Precedent, 2020. Available: https://www.nytimes.com/ 2020/01/22/world/asia/coronavirus-quarantines-history.html

7 Hauck G, Gelles K, Bravo V, et al. Five months in: a timeline of how COVID-19 has unfolded in the US, 2020. Available: https://www. usatoday.com/in-depth/news/nation/2020/04/21/coronavirusupdates-how-covid-19-unfolded-u-s-timeline/2990956001/

8 World Health Organization. Timeline of WHO's response to COVID-19, 2020. Available: https://www.who.int/news-room/detail/ 29-06-2020-covidtimeline

9 The White House. Proclamation on Declaring a national emergency concerning the novel coronavirus disease (COVID-19) outbreak, 2020. Available: https://www. whitehouse.gov/presidential-actions/ proclamation-declaring-national-emergency-concerning-novelcoronavirus-disease-covid-19-outbreak/\#: :text=1601\%20et\% 20seq.), \%2C\%20beginning\%20March\%201\%2C\%202020

10 Mervosh S, Lee J, Gamio L, et al. See how all 50 states are reopening New York, NY, 2020. Available: https://www.nytimes.com/ interactive/2020/us/states-reopen-map-coronavirus.html

11 Baumeister RF, Leary MR. The need to belong: desire for interpersonal attachments as a fundamental human motivation. Psychol Bull 1995; 117:497-529.

12 Jetten J, Haslam SA, Cruwys T, et al. Advancing the social identity approach to health and well-being: progressing the social cure research agenda. Eur J Soc Psychol 2017;47:789-802.

13 Williams WC, Morelli SA, Ong DC, et al. Interpersonal emotion regulation: implications for affiliation, perceived support, relationships, and well-being. J Pers Soc Psychol 2018;115:224-54.

14 Dyal SR, Valente TW. A systematic review of loneliness and smoking: small effects, big implications. Subst Use Misuse 2015;50:1697-716.

15 Chou K-L, Liang K, Sareen J. The association between social isolation and DSM-IV mood, anxiety, and substance use disorders: wave 2 of the National epidemiologic survey on alcohol and related conditions. J Clin Psychiatry 2011;72:1468-76.

16 Haslam C, Jetten J, Cruwys T. The new psychology of health: unlocking the social cure. Routledge, 2018.

17 Hawkley LC, Cacioppo JT. Loneliness matters: a theoretical and empirical review of consequences and mechanisms. Ann Behav Med 2010;40:218-27.

18 Luchetti M, Lee JH, Aschwanden $\mathrm{D}$, et al. The trajectory of loneliness in response to COVID-19. Am Psychol 2020;75:897-908.

19 Pearman A, Hughes ML, Smith EL, et al. Age differences in risk and resilience factors in COVID-19-Related stress. J Gerontol B Psychol Sci Soc Sci 2020. doi:10.1093/geronb/gbaa120. [Epub ahead of print: 03 Aug 2020].

20 Barzilay R, Moore TM, Greenberg DM, et al. Resilience, COVID19-related stress, anxiety and depression during the pandemic in a large population enriched for healthcare providers. Transl Psychiatry 2020;10:1-8.

21 Moccia L, Janiri D, Pepe M, et al. Affective temperament, attachment style, and the psychological impact of the COVID-19 outbreak: an early report on the Italian general population. Brain Behav Immun 2020;87:75-9.

22 McGinty EE, Presskreischer R, Han H, et al. Psychological distress and loneliness reported by US adults in 2018 and April 2020. JAMA 2020;324:93.

23 Holingue C, Badillo-Goicoechea E, Riehm KE, et al. Mental distress during the COVID-19 pandemic among US adults without a preexisting mental health condition: findings from American trend panel survey. Prev Med 2020;139:106231.

24 Qiu J, Shen B, Zhao M, et al. A nationwide survey of psychological distress among Chinese people in the COVID-19 epidemic: implications and policy recommendations. Gen Psychiatr 2020;33:e100213.

25 Wang C, Pan R, Wan X, et al. A longitudinal study on the mental health of general population during the COVID-19 epidemic in China. Brain Behav Immun 2020;87:40-8.

26 Yao H, Chen J-H, Xu Y-F. Patients with mental health disorders in the COVID-19 epidemic. Lancet Psychiatry 2020;7:e21. 
27 Bo H-X, Li W, Yang Y, et al. Posttraumatic stress symptoms and attitude toward crisis mental health services among clinically stable patients with COVID-19 in China. Psychol Med 2020:1-2.

28 Munoz-Navarro R, Cano-Vindel A, Schmitz F, et al. Emotional distress and associated sociodemographic risk factors during the COVID-19 outbreak in Spain. medRxiv 2020.

29 Conversano C, Di Giuseppe M, Miccoli M, et al. Mindfulness, age and gender as protective factors against psychological distress during Covid-19 pandemic. Front Psychol 2020;11:1900.

30 Petzold MB, Bendau A, Plag J, et al. Risk, resilience, psychological distress, and anxiety at the beginning of the COVID-19 pandemic in Germany. Brain Behav 2020;10:e01745.

31 Pierce $\mathrm{M}$, Hope $\mathrm{H}$, Ford T, et al. Mental health before and during the COVID-19 pandemic: a longitudinal probability sample survey of the UK population. Lancet Psychiatry 2020;7:883-92.

32 French I, Lyne J. Acute exacerbation of OCD symptoms precipitated by media reports of COVID-19. Ir J Psychol Med 2020:1-4.

33 Verma S, Mishra A. Depression, anxiety, and stress and sociodemographic correlates among General Indian public during COVID-19. Int J Soc Psychiatry 2020;66:756-62.

34 Horesh D, Kapel Lev-Ari R, Hasson-Ohayon I. Risk factors for psychological distress during the COVID-19 pandemic in Israel: loneliness, age, gender, and health status play an important role. $\mathrm{Br} J$ Health Psychol 2020;25:925-33.

35 Sun N, Wei L, Shi S, et al. A qualitative study on the psychological experience of caregivers of COVID-19 patients. Am J Infect Control 2020;48:592-8

36 Luetke M, Hensel D, Herbenick D, et al. Romantic relationship conflict due to the COVID-19 pandemic and changes in intimate and sexual behaviors in a nationally representative sample of American adults. J Sex Marital Ther 2020;46:747-62.

37 Meyer VM, Benjamens S, Moumni ME, et al. Global overview of response rates in patient and health care professional surveys in surgery: a systematic review. Ann Surg 2020. doi:10.1097/ SLA.0000000000004078. [Epub ahead of print: 15 Sep 2020].

38 Kristan J, Suffoletto B. Using online crowdsourcing to understand young adult attitudes toward expert-authored messages aimed at reducing hazardous alcohol consumption and to collect peerauthored messages. Trans/ Behav Med 2015;5:45-52.

39 Mason W, Suri S. Conducting behavioral research on Amazon's mechanical Turk. Behav Res Methods 2012;44:1-23.

40 Briones EM, Benham G. An examination of the equivalency of self-report measures obtained from crowdsourced versus undergraduate student samples. Behav Res Methods 2017:49:320-34.

41 Greenlaw C, Brown-Welty S. A comparison of web-based and paperbased survey methods: testing assumptions of survey mode and response cost. Eval Rev 2009;33:464-80

42 Qualtrics [software]. Provo, UT2020.

43 Chan C, Holosko MJ. An overview of the use of mechanical Turk in behavioral sciences: implications for social work. Research on Social Work Practice 2015;26:441-8.

44 Rural Health Research Center. Rural-Urban Commuting areas (RUCAs), 2007. Available: http://depts.washington.edu/uwruca/rucarural.php

45 Meilleur A, Subramanian SV, Plascak JJ, et al. Rural residence and cancer outcomes in the United States: issues and challenges. Cancer Epidemiol Biomarkers Prev 2013;22:1657-67.
46 Hart LG, Larson EH, Lishner DM. Rural definitions for health policy and research. Am J Public Health 2005;95:1149-55.

47 Little RJA. A test of missing completely at random for multivariate data with missing values. J Am Stat Assoc 1988;83:1198-202.

48 Singer J, Willett J. Applied longitudinal data analysis: modeling change and event occurrence. USA: Oxford University Press, 2003.

49 Hedeker D, Gibbons RD. Longitudinal data analysis. Hoboken, NJ: John Wiley and Sons, 2006.

50 Newman DA. Missing data: five practical guidelines. Organizational Research Methods 2014:17:372-411.

51 Tennant PW, Harrison WJ, Murray EJ, et al. Use of directed acyclic graphs (DAGs) in applied health research: review and recommendations. medRxiv 2019.

52 Braun V, Clarke V. Using thematic analysis in psychology. Qual Res Psychol 2006;3:77-101.

53 Eysenbach G. Improving the quality of web surveys: the checklist for reporting results of Internet E-Surveys (cherries). J Med Internet Res 2004;6:e34

54 Leach LS, Butterworth P, Poyser C, et al. Online recruitment: feasibility, cost, and representativeness in a study of postpartum women. J Med Internet Res 2017;19:e61.

55 Knack JM, Chen Z, Williams KD, et al. Opportunities and challenges for studying disaster survivors. Analyses of Social Issues and Public Policy 2006;6:175-89.

56 Koch NS, Emrey JA. The Internet and opinion measurement: surveying marginalized populations. Soc Sci Q 2001:82:131-8.

57 Merikangas K, Milham M, Stringaris A. The coronavirus health impact survey (crisis) V0.3, 2020. Available: https://github.com/nimh-mbdu/ CRISIS

58 Warttig SL, Forshaw MJ, South J, et al. New, normative, Englishsample data for the short form perceived stress scale (PSS-4). $J$ Health Psychol 2013;18:1617-28.

59 Cella D, Riley W, Stone A, et al. The patient-reported outcomes measurement information system (PROMIS) developed and tested its first wave of adult self-reported health outcome item banks: 20052008. J Clin Epidemiol 2010;63:1179-94.

60 Hunt SM, McEwen J, McKenna SP. Measuring health status: a new tool for clinicians and epidemiologists. J R Coll Gen Pract 1985;35:185-8

61 Bush K, Kivlahan D, McDonell M. The audit alcohol consumption questions (AUDIT-C): an effective brief screening test for problem drinking. Arch Intern Med 1998;158:1789-95.

62 Global Adult Tobacco Survey Collaborative Group. Tobacco questions for surveys: a subset of key questions from the global adult tobacco survey (GATS). Atlanta, GA: Centers for Disease Control and Prevention, 2011

63 Jensen RE, King-Kallimanis BL, Sexton E, et al. Measurement properties of PROMIS sleep disturbance short forms in a large, ethnically diverse cancer cohort. Psychological Test and Assessment Modeling 2016;58:353-70.

64 Hagen KB, Aas T, Lode K, et al. Illness uncertainty in breast cancer patients: validation of the 5-item short form of the Mishel uncertainty in illness scale. Eur J Oncol Nurs 2015;19:113-9.

65 Carver CS. You want to measure coping but your protocol's too long: consider the brief cope. Int J Behav Med 1997;4:92-100.

66 Bodenmann G, Arista LJ, Walsh KJ, et al. Dyadic coping inventory. In: Encyclopedia of couple and family therapy. Cham: Springer, 2018: $1-5$. 